\title{
MAGNETRON SPUTTERING WITH HOT SOLID TARGET: THERMAL PROCESSES AND EROSION
}

\author{
Alena O. Borduleva*, Galina A. Bleykher, Dmitrit V. Sidelev, \\ VALERIY P. KRIVOBOKOV \\ Institute of Physics and Technology, Tomsk Polytechnic University, Lenin Avenue 2a, Tomsk 634028, Russia \\ * corresponding author: tcybenkoao@gmail.com
}

\begin{abstract}
This work focuses on erosion and thermal processes taking place on the surface of the titanium target in magnetron sputtering. The study was carried out using magnetron sputtering systems (MSS) with different thermal insulation target types from the magnetron body. It was found that the presence of an evaporation component allows the rate of removal of atoms from the surface of a solid target to be increased with limited thermal conduction. A mathematical simulation was used to evaluate the contribution of evaporation to the increase in the coating deposition rate for complete and partial thermal insulation.

It was found that non-uniformity of the direct-axis component of the magnetic induction vector helps to localize the heating. also increases the evaporation rate on the surface of the target. It was proved that local evaporation including sublimations on the surface of a hot target is a significant factor in increasing the coating deposition rate. Due to this mechanism, the coating deposition rate can be increased 5 times for $\mathrm{Ti}$ in comparison with fully cooled targets. This result can be applied for direct current magnetrons and also for pulsed systems. It was also found that evaporation increased the energy efficiency of the target erosion. The most suitable metals were selected for obtaining high-intensity emission of atoms from a solid target.
\end{abstract}

KEYWORDS: magnetron sputtering; hot target; evaporation; high rate deposition.

\section{INTRODUCTION}

In recent decades, magnetron sputtering has been considered as one of the most effective methods for depositing high-quality functional coatings on a solid surface. The deposition is carried out in a vacuum diode system under conditions of crossed electric and magnetic fields. The gas discharge plasma that is formed is located close to the surface of the target, which is fixed to the cathode. Positively charged ions extracted from the plasma move to the cathode and accelerate to energies from 100 to $1000 \mathrm{eV}$ in the area of a potential fall. On the surface of the target, the ions transfer their energy to atoms on the surface layer, make the surface layer sputtered and initiate a secondary emission of electrons, which are necessary to maintain the discharge. The flow of sputtered atoms moves mainly forward from the surface of the target. The particles of this flow reach the substrate surface and are deposited on it, forming a coating.

Due to the magnetic field near the surface of the target, the flux density of the ions that bombard the target is is much greater than for simple diode systems. The ion flux density is proportional to the direct-axis component of a magnetic induction vector.

The sputtering rate of magnetron sputtering systems (MSS) is crucial for the whole technology, since it determines its productivity and depends linearly on the ion current density [1.

Unfortunately, the productivity of modern MSS with solid targets often does not meet industrial needs. Attempts to improve their efficiency just by increasing the current density have turned out to be ineffective, since the sputtering rate depends linearly on the current density. For high-power pulsed MSS, the issue related to the increase in the coating deposition rate is further aggravated by intense ionization of the sputtered particles and by the return of the particles to the target [2].

However, this situation might be improved by adding evaporation to sputtering. Previous research has proved that this method, aimed at increasing the removal rate of particles from the surface of target (or its erosion) during MSS operation [3-5] is promising. For this purpose, certain conditions need to be provided, so that the thermal energy supplied to the target from the plasma can be retained in it, and can heat the target to a high temperature. In conventional magnetrons, the target has a very good heat-conducting contact with an intensely cooled magnetron body, into which magnets are placed. As a rule, its temperature is therefore low.

If the structure of the magnetron is changed so that the flow of heat from the target is decreased, the target can be warmed up strongly. In other words, it will become possible for MSS to operate with a "hot target". Moreover, for some materials, significant evaporation can be created by keeping most of the target in the solid state. 


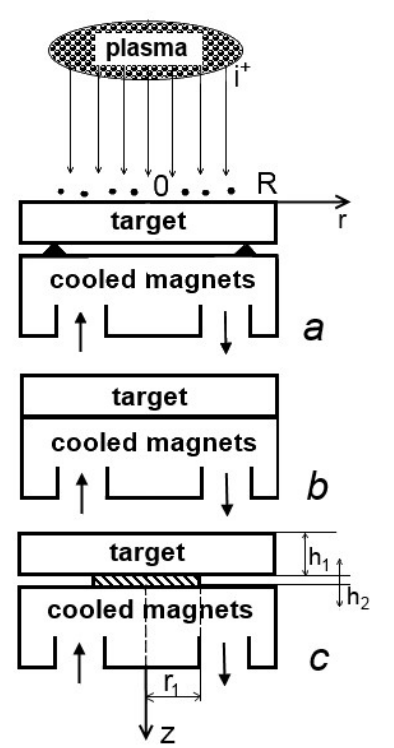

FiguRE 1. Magnetron sputtering scheme with different types of heat conducting contact between the target and the cooled magnetron body: (a) total heat conductivity insulation, when the target is fixed to the cathode by thin insulating insertions, (b) complete cooling, (c) partial heat insulation, when the magnetron body has a small spacer disk in the center to which the target is fixed.

A scheme of magnetron sputtering with different types of heat conductive contact of the target and the magnetron body is shown in Figure 1.

Examples of magnetron sputtering with the implementation of hot targets for increasing the coating deposition rate have been published in the scientific literature [6-8]. This effect occurs not only because of the increase in the surface erosion rate, but also due to other processes such as discharge stabilization in the presence of a hot target during coating deposition when reactive gases are used [8].

It should be noted that the role of the high temperature of the target and of the evaporation from its surface in the formation of the coatings during the operation of different types of MSS has not been studied in full. Our hypothesis is that advances in the solution of this task will contribute to the further development of high-rate magnetron coating deposition technologies, including such promising areas as high power impulse magnetron sputtering.

The purpose of the research presented in this paper was to reveal the regularities of heat and erosion processes in a solid target with partial or complete heat conductive insulation in dependence on the power parameters of pulse magnetron sputtering systems, and also to evaluate the contribution of evaporation to an increase in the coating deposition rate. In addition, a mathematical simulation was applied to solve this task. The adequacy of the model that has been developed was tested by comparing the calculations with empirical data.

\section{A THEORETICAL DESCRIPTION AND MODELING REPRESENTATIONS}

Surface sputtering of a magnetron target is the result of an elastic interaction between high-energy ions from plasma and atoms of the target material. The sputtering rate is equal to the product of the ion flux density and the sputtering yield.

During MSS operation, the energy of the ions bombarding the target does not, as a rule, exceed several $\mathrm{keV}$. According to Peter Sigmund's classification of sputtering modes [1], this is the primary knock-out mode. His formula for this case can therefore be used to calculate the sputtering yield [1]. The sputtering rate $V_{\text {sput }}$ will therefore be expressed by the following expression [4]:

$$
V_{\mathrm{sput}}=\frac{3 \alpha M_{1} M_{2} W_{\mathrm{ion}}}{\pi^{2}\left(M_{1}+M_{2}\right)^{2} n_{0} U_{S}},
$$

where $M_{1}$ and $M_{2}$ refer to the atomic masses of the incident particle and the target material; $\alpha$ is a parameter that depends on the mass relation $M_{2} / M_{1} ; U_{S}$ is the target surface binding energy; $W_{\text {ion }}$ is the ion current power density; and $n_{0}$ is the nuclear density of the target material.

Investigations carried out by various authors have shown that the effect of temperature on the sputtering yield and rate is negligible 912 . It was therefore not taken into account in the calculations.

Sublimation and evaporation have a different nature from sputtering. Since sublimation and evaporation are almost identical, the term "evaporation" will be used throughout the paper in reference to both processes. Their intensity is determined by the surface temperature of the target.

In a vacuum, the evaporation rate is described by the Hertz-Knudsen equation [13]:

$$
V_{\mathrm{ev}}(T)=\frac{1}{n_{0} \sqrt{2 \pi m k T}}\left(p_{\mathrm{sat}}(T)-P^{*}\right),
$$

where $m$ is the mass of an evaporated atom (molecule), $k$ is the Boltzmann constant, $p_{\text {sat }}(T)$ is the saturated vapor pressure at surface temperature $T$, and $P^{*}$ is the hydrostatic pressure above the evaporation surface.

The $P^{*}$ value includes the pressure of the evaporated material above the surface and the working gas pressure in the vacuum chamber.

Thus, in order to determine the evaporation rate on the surface of the MSS target, its temperature needs to be calculated.

The spatial and temporal temperature distribution depending on the MSS parameters is also of interest, because the heat radiation flux can have a significant influence on the properties of the deposited coating and the treated workpiece.

For this purpose, the heat conductivity equation written in the cylindrical coordinate system with az- 
imuthal symmetry was used:

$$
\begin{array}{r}
\frac{\partial E(z, r, t)}{\partial t}-V(t) \frac{\partial E(z, r, t)}{\partial z}=\lambda\left(\frac{\partial^{2} T(z, r, t)}{\partial z^{2}}\right. \\
\left.+\frac{1}{r} \frac{\partial T(z, r, t)}{\partial r}+\frac{\partial^{2} T(z, r, t)}{\partial r^{2}}\right),
\end{array}
$$

where $V(t)=V_{\text {sput }}(t)+V_{\mathrm{ev}}(t)$ is the total rate of removal of particles from the surface of the target, or the erosion rate.

The boundary condition on the treated surface is as follows:

$$
\begin{aligned}
\left.\lambda \frac{\partial T(z, r, t)}{\partial z}\right|_{\mathrm{z}=0}=-q(r, t)+Q_{\mathrm{ev}}(r, t) \\
\quad+Q_{\mathrm{rad}}(r, t)+Q_{\mathrm{sput}}(r, t)+Q_{\mathrm{ref}}(r, t) .
\end{aligned}
$$

The initial conditions are:

$$
T(z, r)=T_{0}(z, r) .
$$

The $O z$ axis is perpendicular to the treated surface, along the direction of the falling ion flux; the $O r$ axis is directed along the surface (Figure 1).

$E(z, r, t)$ is the function of the thermal component of the internal energy, and $T(z, r, t)$ is the temperature in the "target in the crucible" system; $\lambda$ is the heat conductivity coefficient.

In (4), $q(r, t)$ is the power density of the plasma acting on the surface of the target. Our previous studies show that this value is almost the same as the power density of the ion flux bombarding the surface of the target, i.e. $W_{\text {ion }}$ 14. The variation of $W_{\text {ion }}$ in time is defined by the power oscillogram of the magnetron energy supply. The $W_{\text {ion }}$ distribution along the surface of the target corresponds to the spatial distribution of the direct-axis component of the magnetic induction vector near it $\left(B_{r}(r)\right) . B_{r}(r)$ does not usually change over time, so the $W_{\text {ion }}(r, t)$ function can be represented as follows:

$$
W_{\text {ion }}(r, t)=\frac{P_{\text {ion }}(t) B_{r}(r)}{2 \pi \int_{0}^{R} r B_{r}(r) \mathrm{d} r},
$$

where $P_{\text {ion }}(t)$ is the total power of the ion current of the magnetron discharge, which in general changes over time, and $R$ is the radius of the target.

Ion energy is spent on target sputtering, heating, melting and evaporation. Some proportion of the high energy particles and, consequently, their energy, is reflected from the irradiated surface. The heat radiation from strongly heated target surfaces should also be taken into account. The following energy losses from the treated target surface are therefore presented in (4): $Q_{\mathrm{ev}}$ stands for evaporation, $Q_{\mathrm{rad}}$ is heat radiation, $Q_{S}$ is sputtering, and $Q_{\text {ref }}$ is the energy of the reflected particles.

On the lateral surfaces of the target, the heat is passed into the environment due to heat radiation. Particular attention should be paid to the boundary condition on the back surface of the target, which contacts the cooled magnetron body. There are two cases of heat exchange with the environment. In the case when there is no direct contact of the back surface of the target with the cooled magnetron body, the energy loss due to heat radiation is specified. In the contact area, the condition of heat conduction flux continuity is accepted.

Solving this task enables a calculation to be made of the temperature of the target and of the atom removal rate from any element on its surface at any given time, using DC and repetitively-pulsed MSS power supplies.

\section{Results And Discussion}

The calculations were carried out for planar magnetrons, which operate in repetitively-pulsed power mode with a pulse repetition frequency of $1 \mathrm{kHz}$. The average power in a pulse was from $10^{2}$ to $10^{4} \mathrm{~W}$, which corresponds to power density transferred to the target from 10 to $10^{3} \mathrm{~W} / \mathrm{cm}^{2}$. The working gas was argon.

The dependence of the ion current power on time $W_{\text {ion }}(t)$ repeats the change in discharge power $Q(t)=$ $U(t) I(t)$, where $U$ refers to the voltage and $I$ and refers to the current of the discharge. Their change during a pulse of duration $\tau$ is determined by the type of power supply. For a repetitively-pulsed impact, the average values of these quantities should be taken into account. In this work, two types of power and ion current power density averaging were used: averaging over pulse $W_{\text {imp }}=\int_{0}^{\tau} W_{\text {ion }} \mathrm{d} t$ and averaging over period $W_{\text {per }}=\tau \nu W_{\text {imp }}=\delta W_{\text {imp }}$, where $\delta$ is the duty cycle, and $\nu$ is the pulse repetition frequency.

Considering the effect of temperature on the intensity of the MSS target surface erosion, the question arises of the evolution of the heating up and the impact of the power supply on this process.

Our calculations have shown that in repetitivelypulsed mode with period averaged power density $W_{\text {per }} \leq 10^{3} \mathrm{~W} / \mathrm{cm}^{2}$, even in the case of complete heat target insulation, the heating takes place gradually while the heat energy is accumulating from the plasma. At first, the temperature increases rapidly. After that, it slows down due to an increase in energy losses due to heat radiation. A balance between incoming and outgoing power is established. The thermal field in the target becomes stationary and is determined by period averaged power. The pulse repetition frequency and the power during the individual pulses do not influence this valuethe period averaged power. The results for erosion enhancement due to evaporation obtained for the period averaged power can therefore be applied for MSS with a different power supply (DC, mid-frequency and high-frequency, and high-power pulsed magnetron sputtering).

When the temperature stabilizes, the evaporation rate remains almost constant throughout the current period, because the surface temperature of the target does not have time to change significantly between two adjacent pulses (Figure 2). The sputtering rate follows the current variation in time. Therefore, the erosive 

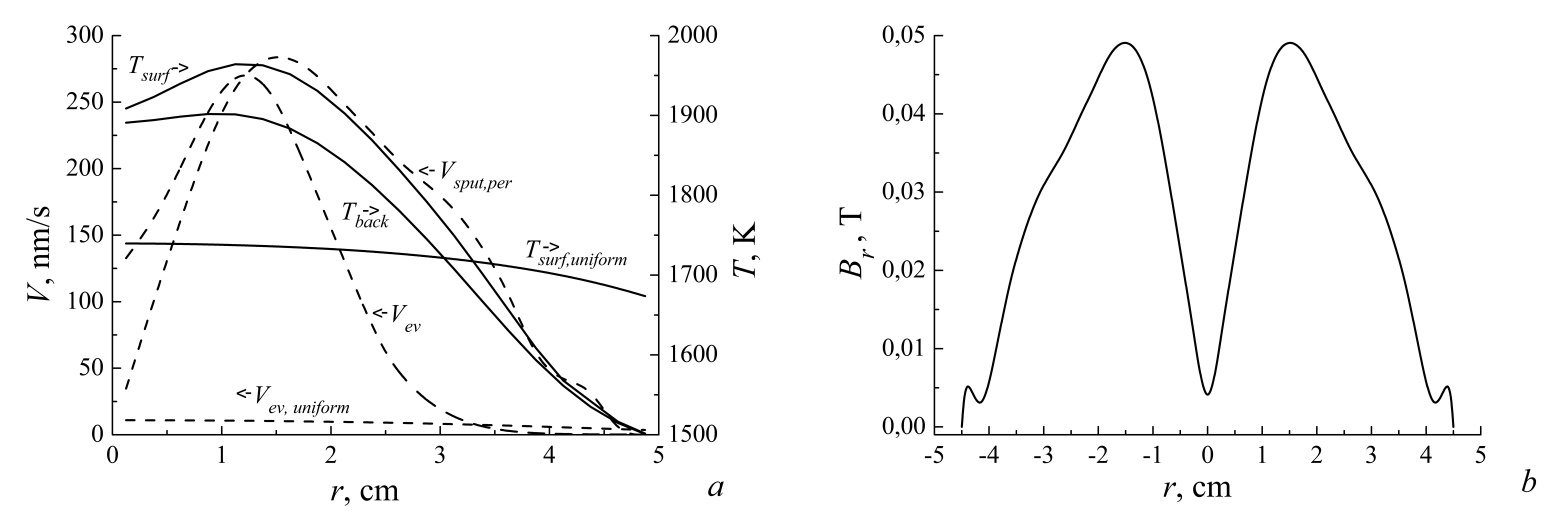

Figure 3. Temperature radial distribution $\left(T_{\text {surf }}-\right.$ on the treated surface; $T_{\text {back }}$ - on the surface of the rear side), evaporation rate $\left(V_{\mathrm{ev}}\right)$ and sputtering rate averaged over the period $\left(V_{\text {sput,per }}\right)$ of the heat insulated Ti target in the stage of stabilization of MSS operation with power $1900 \mathrm{~W}$ averaged over the period (a); distribution of the direct-axis component of magnetic induction vector $B_{r}(\mathrm{~b})$. The target has a radius of $5 \mathrm{~cm}$, and is $0.6 \mathrm{~cm}$ in thickness.

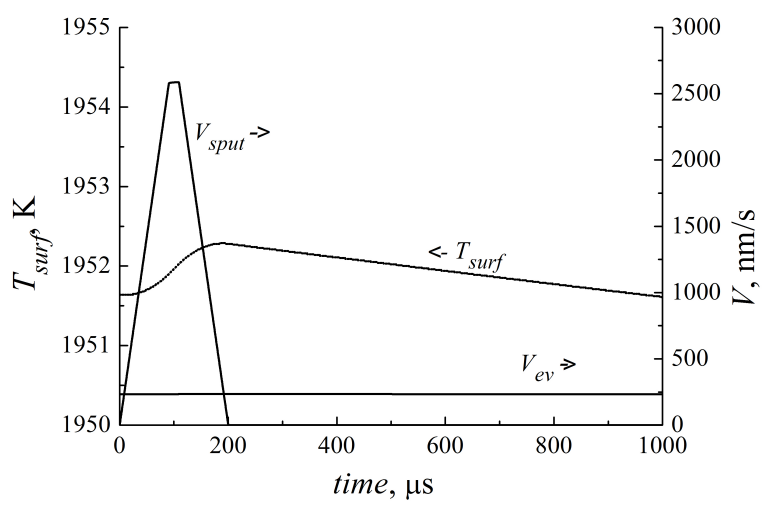

Figure 2. Maximum temperature evolution on the surface of the insulated Ti target $\left(T_{\text {surf }}\right)$, evaporation rate $\left(V_{\text {ev }}\right)$ and sputtering rate $\left(V_{\text {sput }}\right)$ during one period of the pulsed MSS $\left(W_{\text {per }}=24.2 \mathrm{~W} / \mathrm{cm}^{2}\right.$, $\nu=1 \mathrm{kHz}, \delta=20 \%$ ) in steady state mode. The target has a radius of $5 \mathrm{~cm}$, and is $0.6 \mathrm{~cm}$ in thickness.

flow from the surface of the target is a continuous flow of vaporized particles on the background of which sputtered atoms appear periodically. For example, Figure 2 presents the situation during the current period at a pulse repetition frequency of $\nu=1 \mathrm{kHz}$, duty cycle $\delta=20 \%$ and $W_{\text {per }}=24.2 \mathrm{~W} / \mathrm{cm}^{2}$. The power oscillogram is close to trigonal in shape. In this case, the evaporation rate Vev almost coincides with the period averaged sputtering rate $\left(V_{\text {sput,per }}\right)$. However, the sputtering rate during the current pulse is proportional to the instantaneous power density, and is significantly greater than $V_{\mathrm{ev}}$.

Evaporated particles leave the target not only during the action of the current pulse, but also between pulses. This should make it possible to enhance the coating deposition rate for high-power pulsed magnetron sputtering. Between the current pulses, there are almost no intensive ionization processes in the gas discharge area. In this case, the probability of the emitted particles reaching the substrate significantly increases. The greater the number of these particles, the higher the coating growth rate should be.

It is known that the sputtering rate is non-uniform along the target surface during MSS operation, because the ion current power density is proportional to the direct-axis component of the magnetic induction vector $\left(B_{r}\right)$.

Calculations show that $W_{\text {ion }}(r)$ non-uniformity also influences the radial distribution of the temperature and evaporation rate. The temperature behavior and the erosion rate components on the surface of the Ti target in steady state magnetron operation are presented in Figure 3, where $r=0$ is the centre of target $r$. They were calculated for the real and the hypothetical uniform distribution of $B_{r}(r)$. Its profile, which we measured for a planar magnetron with permanent magnets, is also shown in Figure $3 \mathrm{~b}$. This is a typical profile for modern MSS. This distribution was used for calculations of the temperature, evaporation and sputtering rates. $T_{\text {surf, uniform }}$ and $V_{\text {ev, uniform }}$ are the temperature and evaporation rates when the ion current density distribution along the target surface is uniform.

Figure 3 shows that non-uniformity of magnetic induction along the target surface is a favorable factor for evaporation, because the heating of local areas increases. This evaporation enhancement apparently should occur for materials with a relatively low thermal conductivity coefficient. Figure $3 \mathrm{~b}$ indicates that the area on the surface of the target where the evaporation rate is highest may be different from the area where the sputtering rate reaches its peak.

Figure 4 shows the dependence of the surface temperature of the Ti target and the coating deposition rate on the period averaged ion current power density. The temperature was calculated on the highest heating area. The deposition rate was calculated for the substrate area opposite to the center of the target. The methodology for calculating the deposition rate 


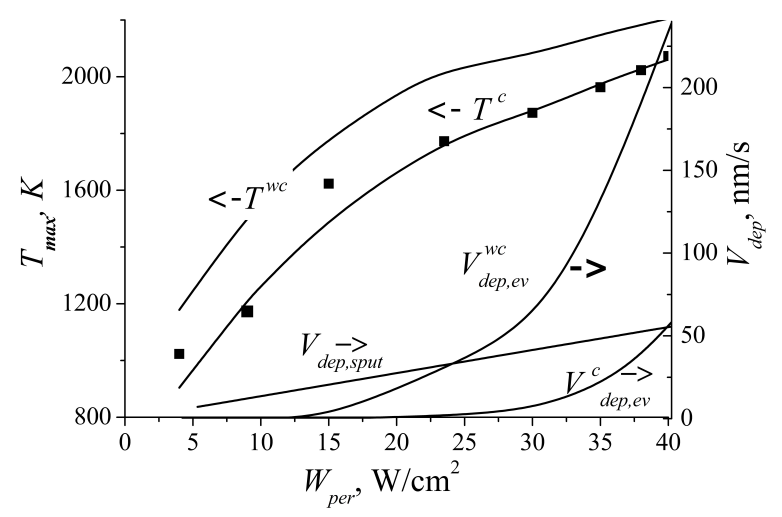

Figure 4. The dependence of the maximum temperature on the surface of the Ti target $\left(T^{w c}\right.$ — without cooling, $T^{c}$ - with partial cooling) and on the coating deposition rate (a point opposite the center of the target at a distance of $8 \mathrm{~cm}$ ) due to sputtered particles $\left(V_{\text {dep,sput }}\right)$ and evaporated particles $\left(V_{\text {dep,ev }}^{w c}-\right.$ without cooling; $V_{\mathrm{dep}, \mathrm{ev}}^{c}$ - with partial target cooling) on the averaged power density $W_{\text {per. }}$. The target has a radius of $5 \mathrm{~cm}$, and is $0.6 \mathrm{~cm}$ in thickness. The cooling is according to Figure 1 , where $r_{1}=0.5 \mathrm{~cm}, h_{2}=0.2 \mathrm{~cm}$; the body and the disk of the magnetron are prepared from copper. The solid lines are for the calculation results; the dots are for the experimental results for $T_{\max }$ from [14, 15].

\section{is described in 5 .}

The results were obtained for the cases of complete insulation of the target (superscript "wc") and partial insulation of the target (superscript "c") in the steady thermal state of MSS operation. The partial insulation parameters correspond to Figure 1p, where the spacer disk radius $r_{1}$ is equal to $0.5 \mathrm{~cm}$, its thickness $h_{2}$ is $0.2 \mathrm{~cm}$, and both the disk and the magnetron body are made of copper.

The surface temperature of the target increases non-linearly with $W_{\text {per }}$ growth. The heat conductivity value is lower than for complete insulation.

The developmental character of the heating processes in the target obtained by calculation was verified by the experimental results published in [15]. The authors measured the temperature evolution on the surface of the Ti target of pulsed MSS with an infrared camera. The power supply frequency was equal to $1 \mathrm{kHz}$ and $10 \mathrm{kHz}$, and the duty cycle was $20 \%$. The measured temperature values are shown as dots in Figure 4 . The good agreement between the calculation results and the experimental results gives reason to believe that our modeling representations of the development of the heating processes in the target of the pulse magnetron are correct.

The data from Figure 4 indicate that at low $W_{\text {per }}$ the target is just sputtered. In this case, the deposition rate is proportional to the power density, and is no more than several $\mathrm{nm} / \mathrm{s}$. The evaporation becomes noticeable at $W_{\text {per }} \approx 17 \mathrm{~W} / \mathrm{cm}^{2}$, when the target is completely heat insulated, and at $28 \mathrm{~W} / \mathrm{cm}^{2}$, when

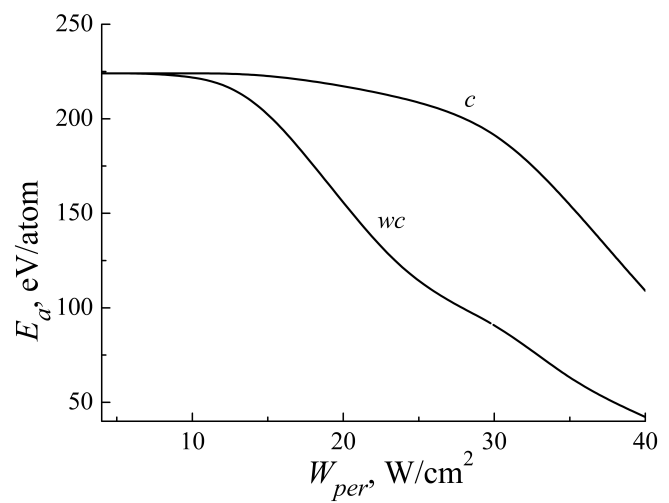

Figure 5. The dependence of the energy that has to be supplied in the $\mathrm{Ti}$ target to remove one atom from its surface $\left(E_{\mathrm{a}}\right)$ on the ion current power density averaged over period ( $W_{\text {per }}$ ) for complete heat conduction insulation ("wc") and for partial heat conduction insulation ("c") of the target.

the target has a partial heat conductive contact with the cooled magnetron body. At $W_{\text {per }} \approx 24.2$ and $40 \mathrm{~W} / \mathrm{cm}^{2}$, the deposition rates due to evaporation and sputtering are approximately the same. At higher power of the magnetron, the evaporation component in the deposited flux rapidly becomes predominant.

The calculations show that the increase in the $\mathrm{Ti}$ coating growth rate because of the evaporation from the hot target can be about five times higher than for usual sputtering with complete cooling of the target.

When there are a significant number of evaporated particles, the dependence $V_{\text {dep }}\left(W_{\text {per }}\right)$ becomes nonlinear. In this case, the amount of energy $E_{\mathrm{a}}$ that should be transferred to the target from the plasma in order to remove an atom from its surface decreases. This parameter was calculated as:

$$
E_{\mathrm{a}}=\frac{W_{\text {per }}}{V_{\text {erosion }}}
$$

Figure 5 presents the dependence of $E_{\mathrm{a}}\left(W_{\text {per }}\right)$ for cases of complete and partial target heat insulation (the partial insulation scheme is similar to the previously described scheme). When there is only sputtering, $E_{\mathrm{a}}$ is equal to $224 \mathrm{eV} /$ atom. The presence of evaporation reduces this value to $42.2 \mathrm{eV} /$ atom, i.e., almost fivefold. The change in the energy losses from the Ti target depending on the period averaged ion current power density is shown in Figure 6 .

The cases of complete insulation and (the scheme described above) are analyzed here. In the case of complete insulation, the main energy loss is through heat radiation within all the possible range of the MSS power density. In the case of partial cooling, the main energy loss at low power is through heat conduction from the target. Then heat radiation becomes predominant. About $0.9 \%$ of the energy from the plasma is spent on sputtering. 


\begin{tabular}{lcccc}
\hline Target material & $W_{\text {ion }}\left(\mathrm{W} / \mathrm{cm}^{2}\right)$ & $T_{\text {surf } \max } / T_{\text {melt }}$ & $V_{\text {ev }}(\mathrm{nm} / \mathrm{s})$ & $V_{\text {sput,per }}(\mathrm{nm} / \mathrm{s})$ \\
\hline $\mathrm{Cr}$ & 12.7 & 0.8 & 190 & 130 \\
$\mathrm{Ti}$ & 24.2 & 1 & 270 & 280 \\
$\mathrm{Zn}$ & 1.66 & 0.9 & 78 & 81 \\
$\mathrm{Cu}$ & 58.6 & 1.2 & 360 & 980 \\
\hline
\end{tabular}

TABle 1. Pure metals that can be used as a hot target in MSS $\left(W_{\text {ion }}\right)$ is ion current power density, $T_{\text {surf, max }} / T_{\text {melt }}$ is the ratio of the maximum target surface temperature to the target melting temperature, $V_{\mathrm{ev}}$ is the evaporation rate, $V_{\text {sput,per }}$ is the period averaged sputtering rate). The cooling system was as in Figure 1 1a. Calculations were carried out at $\nu=1 \mathrm{kHz}, \delta=20 \%$.

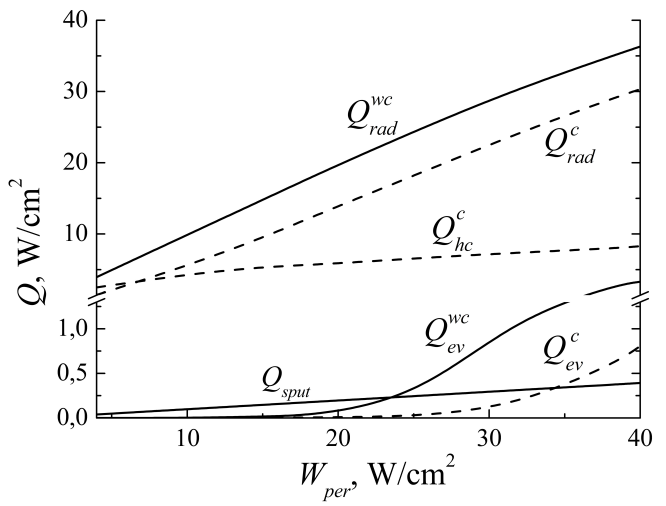

Figure 6. Dependence of the power density of the energy losses from the Ti target for complete heat insulation (superscript "wc") and for partial heat insulation (superscript "c") according to Figure 1; on the ion current power density averaged over the period. $Q_{\mathrm{rad}}$ is heat radiation, $Q_{\mathrm{hc}}$ is heat conduction, $Q_{\mathrm{ev}}$ is energy for evaporation, $Q_{\text {sput }}$ is the energy taken away by sputtered particles. The thickness of the target is $0.6 \mathrm{~cm}$.

The evaporation consumption within the power range suitable for implementing the solid hot target mode does not exceed a few percent. In this way, the mode of erosion from the solid phase is different from the MSS with the liquid phase, when plasma energy consumption by atom evaporation may become predominant [5].

A combination of evaporation and sputtering with the target kept primarily in the solid state is possible only for some materials.

Table 1 presents data for pure metals; they characterize the situation when the evaporation rate on the surface of the target in the steady thermal mode is approximately equal to the period averaged sputtering rate. Here $\mathrm{Vev}$ is the highest value on the surface, and Vsput,per corresponds to the area with the maximum power density. Calculations were carried out for complete heat insulation of the target. Titanium, chromium and zinc are pure metals for which, when they are sputtered from a hot solid target, enhancement of the coating deposition rate due to the evaporating component can be expected. Copper is not suitable for this purpose.

\section{Conclusion}

Local evaporation (including sublimation) on the surface of the MSS solid hot target is a significant factor for enhancing the coating deposition rate. Its contribution is determined by the MSS period averaged power density and by the thermophysical properties of the target. For magnetrons with a completely heat insulated Ti target, a contribution of evaporation that is commensurate with sputtering occurs at about $24 \mathrm{~W} / \mathrm{cm}^{2}$. In this case, the power limit is equal to $40 \mathrm{~W} / \mathrm{cm}^{2}$. Above this limit, the target can melt significantly and lose its shape.

Due to evaporation, the deposition rate of a Ti coating can be increased by about five times in comparison with a completely cooled target.

Creating evaporation on the surface of a high-power pulsed magnetron target by insulating it thermally could be a way to increase the coating deposition rate, due to the fact that it occurs continuously, and the particles removed from the surface between pulses will not be involved in the ionization processes near the target.

It has been found that the non-uniformity of the direct-axis component of a magnetic induction vector is conducive to heating localization and increases the evaporation rate on the surface of the target.

The occurrence of evaporation reduces the plasma energy that is needed to remove one atom from the surface of a solid target. The amount that is required can be about 5 times less than for a completely cooled target.

The main energy flow from a hot solid target is heat radiation. Not more than a few percent of the plasma energy is spent on removing particles from the surface of the target surface, even in the case of complete heat insulation.

\section{ACKNOWLEDGEMENTS}

This research was supported by the Russian Foundation for Basic Research (project 15-08-01632).

\section{REFERENCES}

[1] Sputtering by Particle Bombardment I. Springer Berlin Heidelberg. Edited by Behrisch, R., 1981. DOI:10.1007/3-540-10521-2

[2] Anders, A.: Deposition rates of high power impulse magnetron sputtering: Physics and economics. J. Vac. Sci. Technol. A 28, 783, 2010. DOI:10.1116/1.3299267 
[3] Bleykher, G.A. et al.: Thermal Processes and Emission of Atoms from the Liquid Phase Target Surface of Magnetron Sputtering System. Russ Phys J 58: 431, 2015. DOI:10.1007/s11182-015-0517-6

[4] Bleykher, G.A. et al.: Magnetron Deposition of Coatings with Evaporation of the Target. Tech. Phys. 60: 1790, 2015. DOI:10.1134/S1063784215120026

[5] Bleykher, G.A. et al.: Energy and substance transfer in magnetron sputtering systems with liquid-phase target. Vacuum V. 124: 11-17, 2016. DOI:10.1016/j.vacuum.2015.11.009

[6] Vlček, J. et al.: Pulsed Magnetron Sputtering of Metallic Films Using a Hot Target. In Society of Vacuum Coaters: Technical Conference Proceedings. Santa Clara: Society of Vacuum Coaters: 219-223, 2009. https ://www. scopus.com/record/display.uri?eid= 2-s2.0-77749242269\&origin=inward\&txGid=0

[7] Mercs, D. et al.: Hot target sputtering: A new way for high-rate deposition of stoichiometric ceramic films. Surf. Coat. Technol. 201: 2276-2281, 2006. doi: 10.1016/j.surfcoat.2006.03.035.

[8] Čyvienè, J. et al.: Lithuanian J. Phys. 44: 353-358, 2004.

http://www.itpa.lt/ lfd/Lfz/445/05/Ljp44505.pdf
[9] Behrish, R. et al.: Sputtering yield increase with target temperature for Ar. Nucl. Instr. and Meth. in Phys. Res. B. V. 82: 255-258, 1993. DOI:10.1016/0168-583X(93)96027-A

[10] Coventry, M.D. et al.: Temperature dependence of liquid Sn sputtering by low-energy $\mathrm{He}+$ and $\mathrm{D}+$ bombardment. Journal of Nuclear Materials V. 335: 115-120, 2004. DOI:10.1016/j.jnucmat.2004.06.009

[11] Grossman, A. et al.: Surface tension enhancement of TRIM sputtering yields for liquid metal targets. Journal of Nuclear Materials V. 290-293: 80-84, 2001. DOI:10.1016/S0022-3115(00)00616-4

[12] Bleykher G.A. et al. Model of Metal Surface Erosion under Irradiation by High-Power Pulsed Ion Beams. Journal of Engineering Thermophysics V. 17, Issue 1.: 24-29, 2008. DOI:10.1007/s11823-008-1003-3

[13] Handbook of Thin Film Technology, V. 1. Edited by L. I. Maissel, R. Glang. McGraw-Hill Book Company, 1970.

[14] Bleykher G.A. et al.: Evaporation factor in productivity increase of hot target magnetron sputtering systems. Vacuum V. 132: 62-69, 2016. DOI:10.1016/j.vacuum.2016.07.030

[15] Tesar, J. et al.: On surface temperatures during high power pulsed magnetron sputtering using a hot target. Surface \& Coatings Technology V. 206: 1155-1159, 2011. DOI:10.1016/j.surfcoat.2011.08.005 